\title{
The role of indigenous health workers in promoting oral health during pregnancy: a scoping review
}

\author{
Ariana C. Villarosa 1,2,3,4*, Amy R. Villarosa ${ }^{1,2,3,4}$, Yenna Salamonson ${ }^{2,4,5}$, Lucie M. Ramjann ${ }^{2,4,5}$, Mariana S. Sousa ${ }^{1,3,4,5,6}$,
} Ravi Srinivas ${ }^{1,2,3,4,7}$, Nathan Jones ${ }^{3}$ and Ajesh George ${ }^{1,2,3,4,7}$

\begin{abstract}
Background: Early childhood caries is the most common chronic childhood disease worldwide. Australian Aboriginal and Torres Strait Islander children are twice more likely to develop dental decay, and contributing factors include poor maternal oral health and underutilisation of dental services. Globally, Indigenous health workers are in a unique position to deliver culturally competent oral healthcare because they have a contextual understanding of the needs of the community.

Methods: This scoping review aimed to identify the role of Indigenous health workers in promoting maternal oral health globally. A systematic search was undertaken of six electronic databases for relevant published literature and grey literature, and expanded to include non-dental health professionals and other Indigenous populations across the lifespan when limited studies were identified.

Results: Twenty-two papers met the inclusion criteria, focussing on the role of Indigenous health workers in maternal oral healthcare, types of oral health training programs and screening tools to evaluate program effectiveness. There was a paucity of peer-reviewed evidence on the role of Indigenous health workers in promoting maternal oral health, with most studies focusing on other non-dental health professionals. Nevertheless, there were reports of Indigenous health workers supporting oral health in early childhood. Although some oral health screening tools and training programs were identified for non-dental health professionals during the antenatal period, no specific screening tool has been developed for use by Indigenous health workers.

Conclusions: While the role of health workers from Indigenous communities in promoting maternal oral health is yet to be clearly defined, they have the potential to play a crucial role in 'driving' screening and education of maternal oral health especially when there is adequate organisational support, warranting further research.
\end{abstract}

Keywords: Indigenous, Aboriginal, Health workers, Oral health, Antenatal

\section{Background}

Globally, Indigenous populations experience inequality in health status across the lifespan compared to their nonIndigenous counterparts [1]. While the factors which contribute to these inequities vary across continents, the inherent issues are remarkably similar. They are attributed

\footnotetext{
* Correspondence: a.villarosa@westernsydney.edu.au

${ }^{1}$ Centre for Oral Health Outcomes, Research Translation and Evaluation

(COHORTE), Ingham Institute for Applied Medical Research, Locked Bag 7103, Liverpool BC, NSW 1871, Australia

${ }^{2}$ School of Nursing \& Midwifery, Western Sydney University, Penrith 2751, Australia

Full list of author information is available at the end of the article
}

to a combination of socioeconomic factors, colonisation, globalisation, migration, trans-generational loss of culture and disconnection from the land [2]. Although there have been some improvements in health outcomes in recent years, the gap in Australia remains significant [3].

In Australia, the Aboriginal and Torres Strait Islander people have poorer outcomes in maternal and infant health. A number of reports focusing on Indigenous people in Australia, New Zealand, Canada and the United States have shown that among pregnant women, the rates of gestational diabetes and the risk of developing Type 2 diabetes are higher compared to non-Indigenous women $[4,5]$. 
Further, infants who are born to Australian Aboriginal and Torres Strait Islander mothers are also more likely to be born pre-term and of low birth weight [6] compared to Australians of other descents. In addition, Aboriginal and Torres Strait Islander children are twice as likely to develop early childhood caries (ECC) in their deciduous teeth and caries in their permanent dentition, compared to other children [7]. ECC is the most common chronic childhood disease worldwide and affects various aspects of the child's functioning and quality of life [8]. Although typically overlooked, some evidence supports that maternal oral health may be associated with these health outcomes $[9,10]$.

During pregnancy, physiological factors such as hormonal variations, incidence of nausea and vomiting and dietary changes, increase the risk of pregnant women to dental problems such as dental decay and periodontal diseases $[11,12]$. This is further exacerbated by sub-optimal diabetes control that has been associated with higher salivary glucose secretion, thus contributing to plaque accumulation $[13,14]$. Poor maternal oral health has also been linked with increased adverse birth outcomes such as preterm birth, low infant birth weight and preeclampsia $[15,16]$. Periodontal disease has also been shown to affect glycaemic control and contribute to diabetes complications [17]. Furthermore, maternal dental decay can also contribute to ECC as some babies can acquire their oral flora from carers, especially mothers, in addition to fomites such as feeding utensils which can facilitate bacteria transmission $[18,19]$.

Indigenous Australian pregnant women have a higher prevalence of dental pain relative to the general population and are more likely to avoid certain food due to these problems [20]. In light of the importance of maternal oral health, all pregnant women in Australia are encouraged to have an oral health check and education with their antenatal care provider, including treatment with a dental professional if required [21], similar to the United States, whereby women are advised to consult a dentist early during pregnancy and are provided oral health education, risk assessment and referral during antenatal care [22]. Regrettably, Indigenous mothers are more likely to attend their first antenatal visit later in the pregnancy, and these visits are less frequent than that of non-Indigenous pregnant women [23, 24]. The literature also reports a trend of underutilisation of preventive health services, including dental services $[25,26]$. While financial cost is a main barrier to health service utilisation for this population [27], underlying social and historical factors continue to contribute to their distrust of healthcare professionals and the health system [28].

In view of the current challenges in accessing oral healthcare for Indigenous pregnant women worldwide, it is timely to explore alternative care providers to facilitate dental health-seeking behaviours. Indigenous Health Workers
(IHWs) are well-placed to fill this gap because of their insight into the specific cultural needs and protocols of their communities, the importance of family networks and the impacts of colonisation on their health status and access to health services. IHWs in Australia originated from the Northern Territory during the 1950s where they were initially professionally employed as leprosy health workers, and later segued into their role as medical assistants. During the 1970s, they were recognised as important cultural brokers within their communities [29] as they were able to bridge the disconnect with non-Indigenous health professionals, and facilitate the process of accessing mainstream services by crossing the cultural barriers $[29,30]$. This resulted in the development of the Aboriginal-controlled health services in Australia. In the non-dental health context, IHWs in Australia have been successful in leading culturally-appropriate health promotion and educational activities, in addition to being advocates for Indigenous communities [31-33]. Nevertheless the roles and functions of IHWs are often not well-defined as their roles are usually contingent on the local community's needs, crossing the boundaries of clinicians, social support workers, cultural mentors and managers [29]. Despite the effectiveness in promoting primary healthcare among their Indigenous communities, a paucity in awareness by other healthcare workers, employers and organisations of their broad skillset may have contributed to the limited opportunity for training and career development [29]. Thus, the role of IHWs in promoting oral health for pregnant Indigenous women has received little attention.

\section{Aim}

This review aimed to identify the potential role of IHWs in promoting oral health during the antenatal period. Specifically, this review sought to address the following research areas:

1) The evidence of the role of IHWs in promoting maternal oral health within Indigenous communities.

2) Training programs available to assist IHWs in oral health promotion during the antenatal period.

3) Available oral health screening tools that can be used by IHWs for women during the antenatal period.

\section{Terminology}

In this review, the term Indigenous health worker was used to include health workers from Indigenous communities globally, and referred to persons who work within communities and act as the bridge between consumers and healthcare providers. They facilitate access to health services within communities and assume a role 
in health promotion and provision of culturally sensitive services [34, 35]. The term encompasses other terms like health worker, community health worker, Aboriginal health worker or Aboriginal liaison officer. In Australia, the terms Aboriginal health worker or Aboriginal and Torres Strait Islander health worker are used rather than Indigenous health worker. The term non-dental health professionals, refers to all health professionals other than dental professionals.

\section{Methods}

\section{Study design}

To source the available evidence regarding the role of IHWs in promoting oral health within Indigenous communities, training programs available to perform their role and functions, and the available oral health screening tools, we undertook a scoping review, using the framework described by Arksey and O'Malley [36]. This framework provided the structure to investigate the extent, range and nature of existing research, summarise the evidence, and identify any gaps within the literature. Unlike a systematic review, scoping reviews do not focus on the quality of the research which can significantly limit the type of studies included, enabling a broad range of literature, including reports, clinical guidelines, consensus statements, qualitative and quantitative studies [36]. Another advantage of a scoping review is the iterative process of going back-and-forth and redefining the study aims and search strategies based on the initial findings. This flexibility enables the researcher to gather a broad range of studies to inform the study topic, particularly suitable for topics with limited published evidence in the literature.

\section{Search strategy}

A preliminary search was conducted with the assistance of a librarian for the following databases: CINAHL, Medline (Ovid), PubMed, ProQuest, Scopus and the Australian Public Affairs Information Service - Aboriginal and Torres Strait Islander Subset. We developed an individualised search strategy for each database according to their indexing terms. Boolean operators, truncations and Medical Subject Headings (MeSH) were also included to accommodate variations in spelling and terminology across countries. Reference lists of key articles were also searched for relevant literature. A search for grey literature through government and non-government organisations was also performed. Search strategies were derived from keywords which included: Aboriginal/Indigenous health worker, community health worker, maternal infant care worker, non-dental/oral health professional, oral health, oral hygiene, dental care, training/education program, oral/dental assessment, pregnant, antenatal, perinatal and assessment/ screening tool.

\section{Inclusion and exclusion criteria}

For this review, we selected all articles published up to July 2017 relating to at least one of the research areas. Except for discussion papers, reviews and study protocols, research papers of all other study designs were included. No restrictions were placed on the quality or location of the study, however, studies which focussed on dental professionals promoting oral health among Indigenous pregnant women, and those not published in the English language were excluded.

\section{Study selection and data extraction}

The data were extracted from the selected studies and categorised under the following headings: author/study location; article type; aims; study design; intervention/ program/screening tool; conclusion and focus area. These were subsequently categorised into the three focus areas corresponding to the research question. The first focus area investigated studies where IHWs undertook a role in promoting oral health among pregnant women. The second and third foci explored studies that described any oral health education/training programs and screening tools developed for IHWs in the antenatal setting. If the initial search across all three focus areas revealed limited or no studies, the search was expanded to IHWs promoting oral health in other settings and all non-dental health professionals involved in maternal oral healthcare (Fig. 1).

\section{Results}

Database searches yielded 1640 records. After 1405 duplicates and records screened by titles were excluded, 235 abstracts were screened for relevance; identifying 63 pertinent records. The full texts of the 63 papers were reviewed using inclusion and exclusion criteria. Three government-published documents were also included. Twenty-two articles were identified in this scoping review in relation to the potential role of IHWs in promoting oral health, training programs and potential oral health screening tools that can be used by IHWs for women during the antenatal period (Fig. 1). Studies were based in the United States $(n=7)$, Canada $(n=2)$, Australia $(n=11)$, Turkey $(n=1)$, and India $(n=1)$. These papers were clustered into three broad focus areas: i) role of IHWs in promoting maternal oral health $(n=12)$; ii) antenatal oral health training programs for IHWs $(n=7)$; and iii) potential antenatal oral health screening tools suitable for IHWs $(n=5)$ (Table 1$)$. 


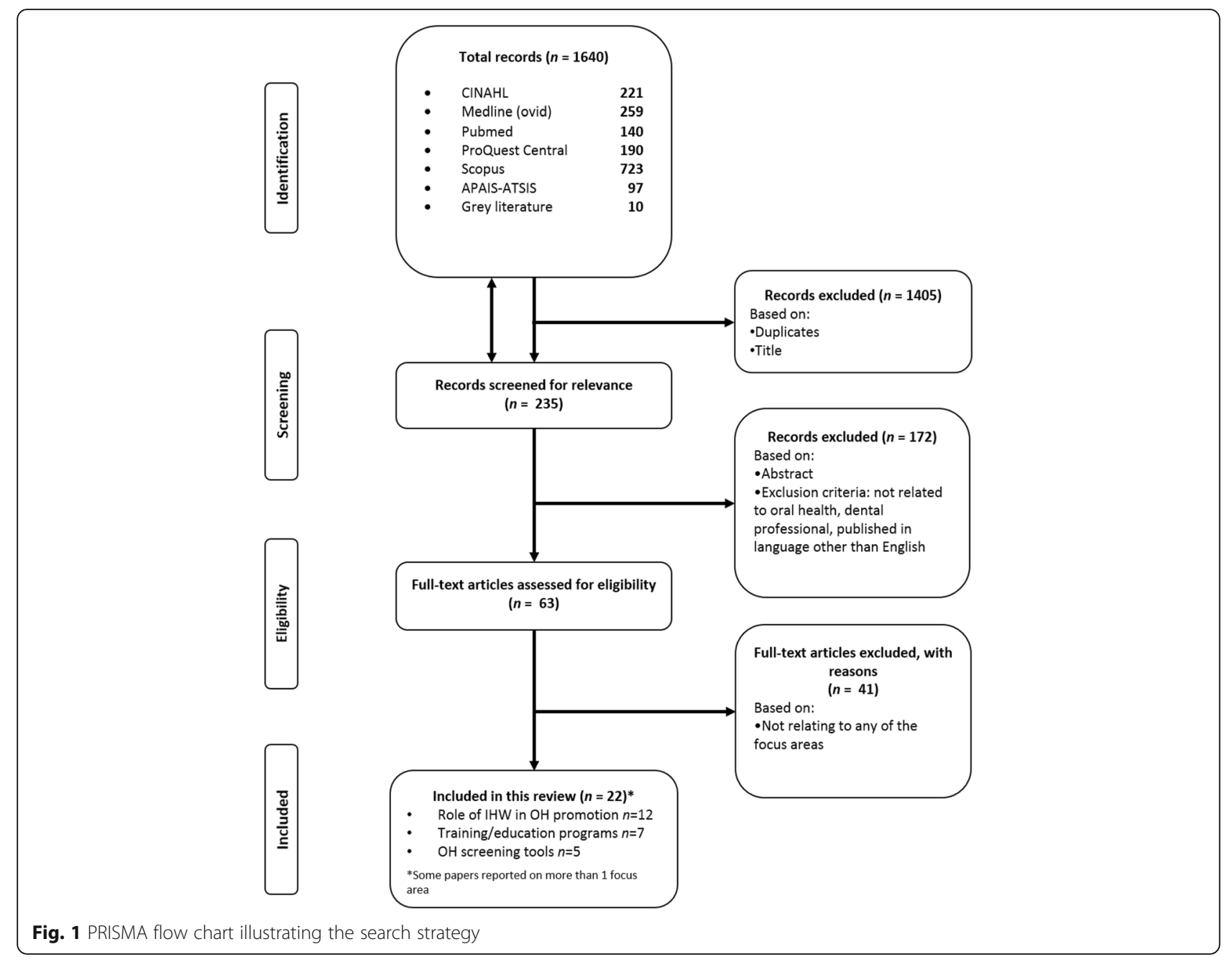

Focus area 1: Role of IHWs in promoting maternal oral health

Within this focus area, three categories of literature were identified that could inform the potential role of IHWs in promoting maternal oral health: i) IHWs who have promoted maternal oral health; ii) IHWs who have supported oral health across the lifespan; and iii) non-dental health professional who have promoted maternal oral health.

\section{IHWs in promoting maternal oral health}

Two articles identified the contribution of IHWs in promoting maternal oral health in an Australian [37] and Canadian context [38]. The Children's Oral Health Initiative (COHI) in Canada aimed to reduce rates of ECC among Indigenous communities through oral health promotion, screening and preventive dental treatment; IHWs were involved in scheduling dental appointments, applying the fluoride varnish and giving one-on-one oral health education to children, expectant mothers and parents in home-based visits. The Aboriginal Oral Health
Program in South Australia [37] developed and streamlined, state referral pathway that involved health workers assessing and referring Australian Aboriginal and Torres Strait Islander pregnant women to dental services. Details of the assessment or their training was not described.

\section{IHWs in supporting oral health across the lifespan}

Five studies reported the role of IHWs in providing oral healthcare for Indigenous populations across the lifespan, ranging from early childhood ( 18 months to 5 years) to elderly [39-43]. Parker et al. [42] evaluated the implementation of a free Indigenous dental clinic in a remote Australian community. Although the roles of IHWs were not clearly defined, they were involved in program development and recruitment. This initiative was well received with a high demand over the year it was funded for implementation. The Dental Health Services in Victoria [41] published a brochure informing clients that free, public dental services were available for Indigenous Australians at a major dental hospital. Aboriginal liaison officers were 


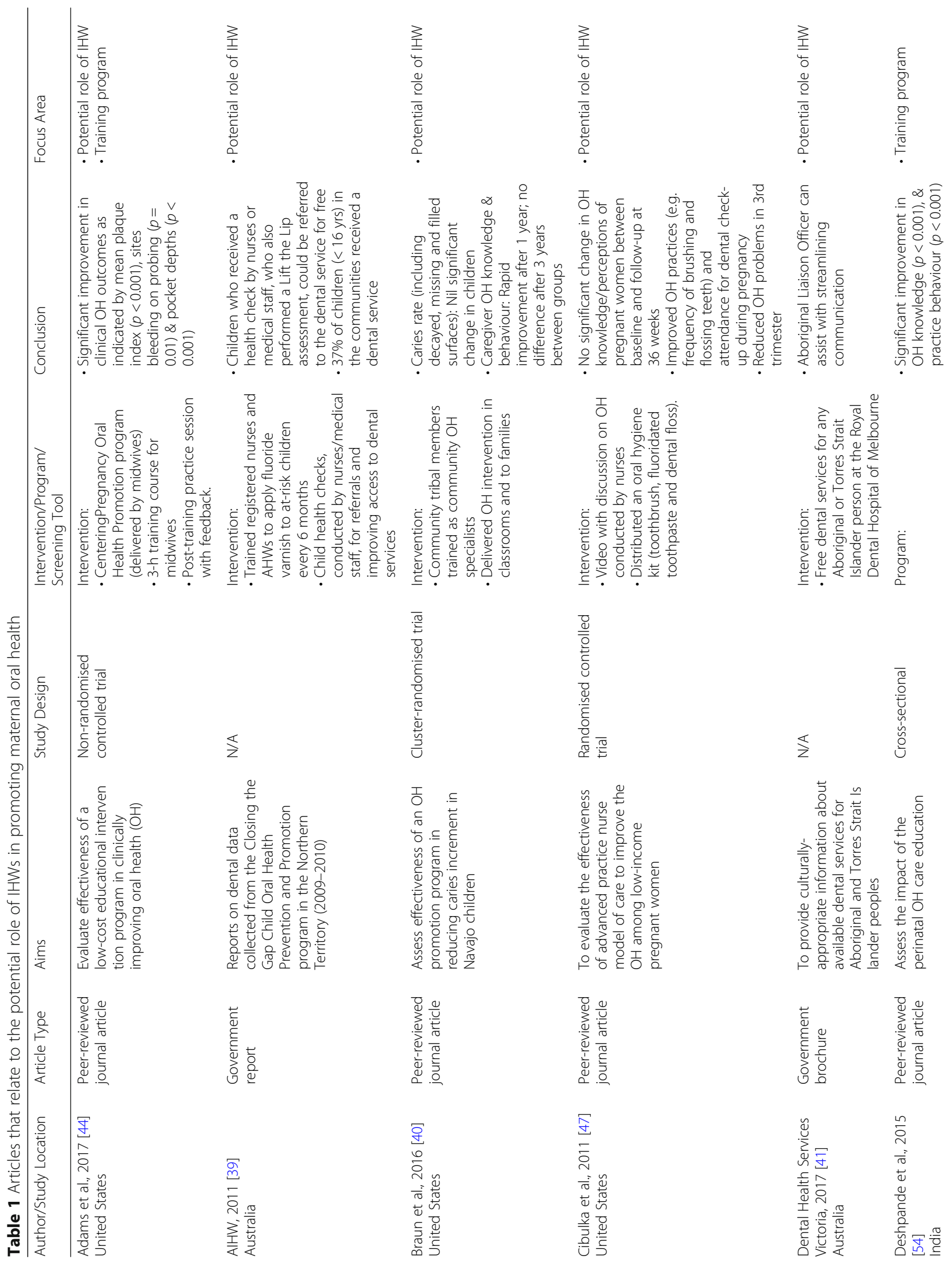




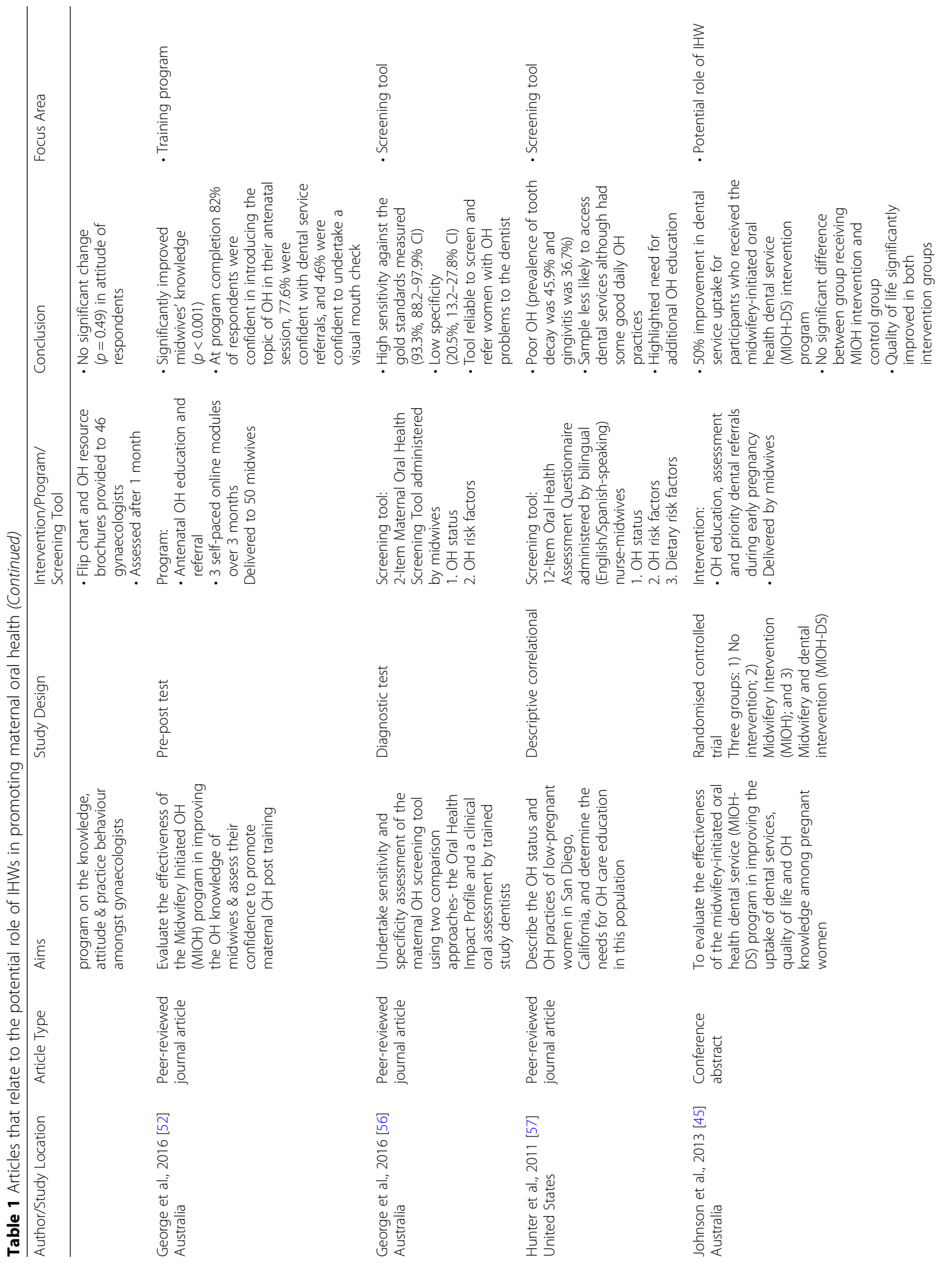




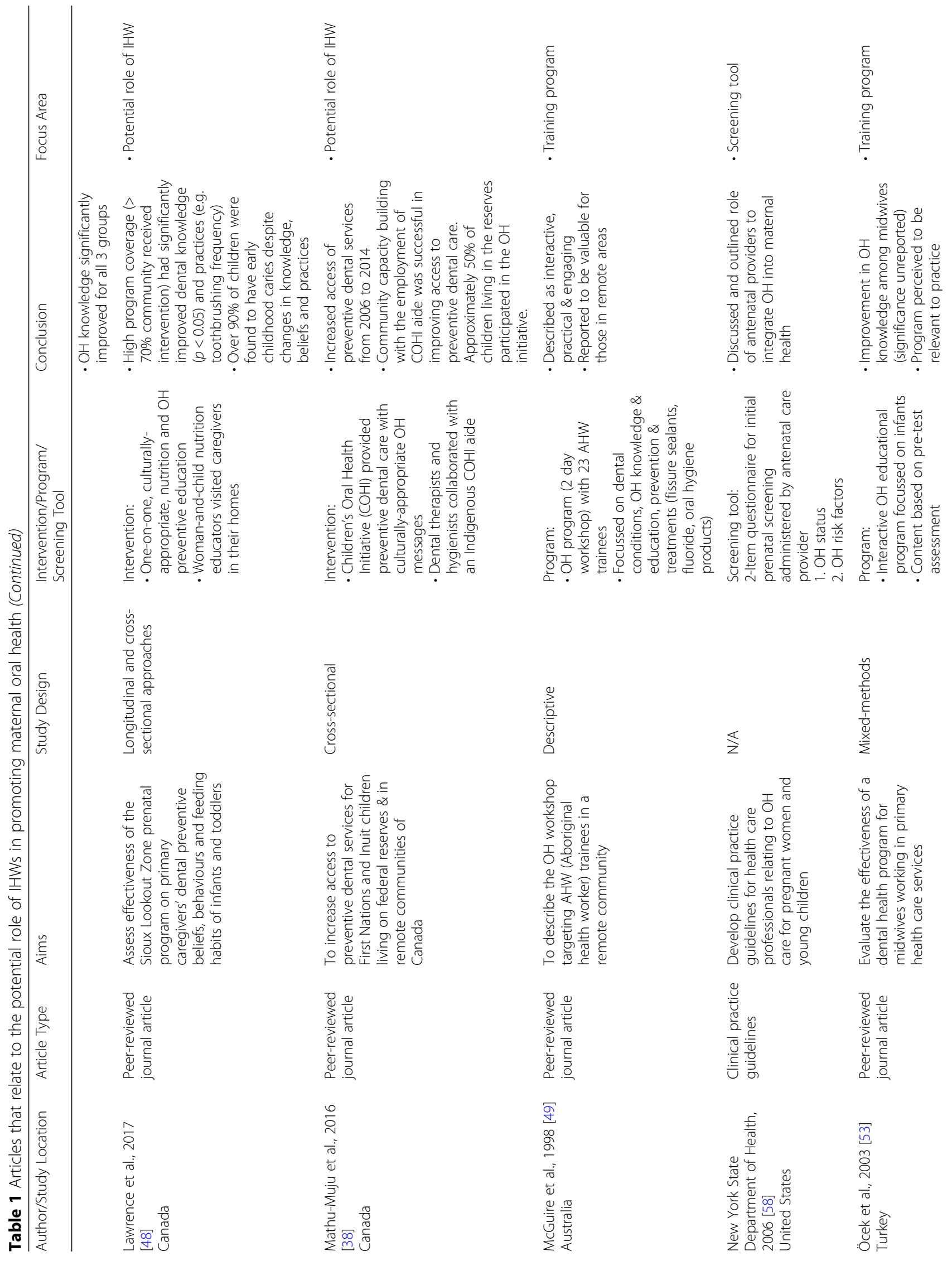




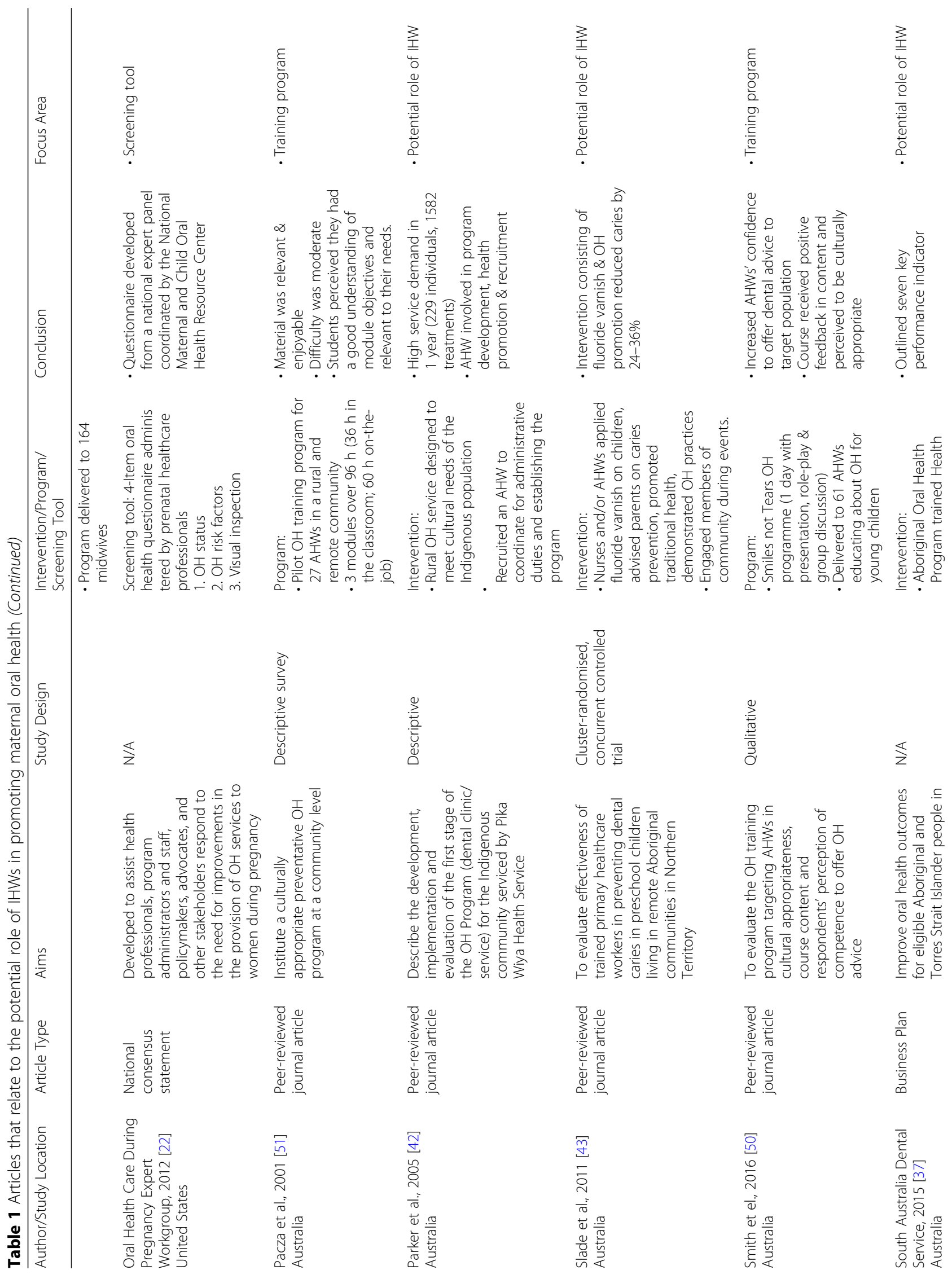




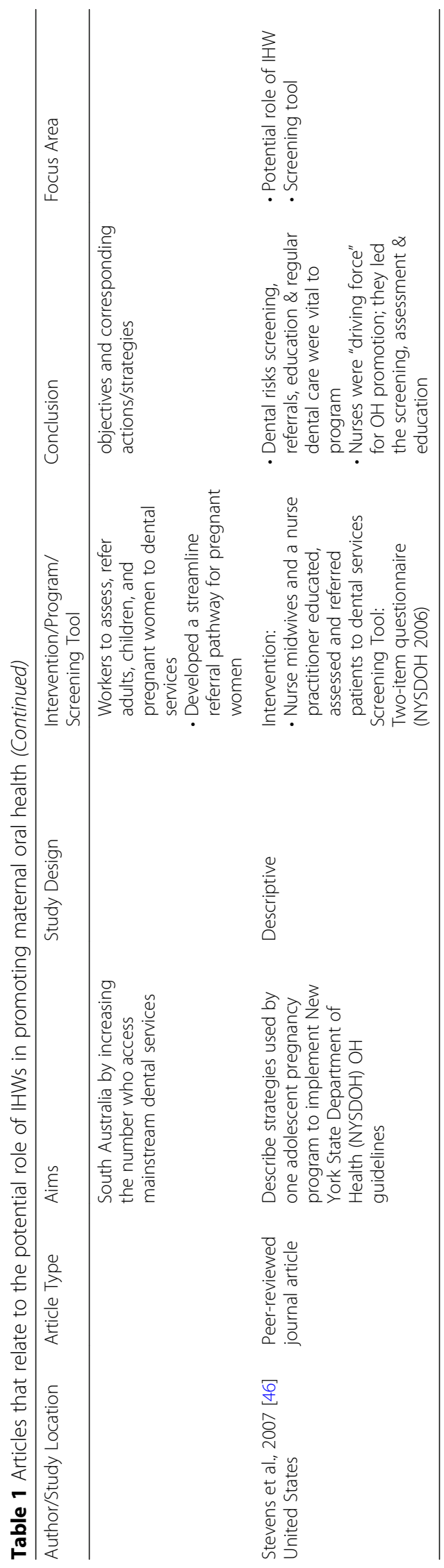


featured as contact personnel to assist in brokering and accessing services. The Australian Institute of Health and Welfare [39] reported that IHWs applied fluoride varnish on children within communities in the Northern Territory and assisted in contacting families as part of the national health campaign, Close the Gap. Among the communities targeted, $37 \%$ of children received a dental service. One Australian [43] and one cluster-randomised trial in the United States [40] also trained IHWs or community tribal members to deliver oral health education and fluoride varnish applications at preschools and within Indigenous communities, respectively. Slade et al. [43] trained Indigenous Australian health workers to assess and refer preschool children with dental decay, and reported reduced incidence of dental caries. No significant change to the children's oral health status as measured by the number of decayed, missing or filled surfaces was reported in the study conducted in the United States [40].

\section{Non-dental health professionals in supporting maternal oral health}

Five studies were identified that described the role of nondental health professionals in promoting maternal oral health, including midwives $[44,45]$, nurses practitioners [46], advance practice nurses [47] and maternal and child nutrition educators [48]. Two studies involving midwives discussed oral health topics with pregnant women, including demonstrating proper oral hygiene techniques, performing oral health screening and providing priority referrals to dental services. In these studies significant improvements were observed in clinical oral health outcomes [44], dental service uptake, self-reported quality of oral health and oral health knowledge of pregnant women. The oral health knowledge and confidence of midwives to promote oral health also showed marked improvement [45]. The New York State Department of Health oral health guidelines, implemented by Stevens et al. [46], described the use of midwives and nurse practitioners to provide oral health screening, education and referrals to dental services; nonetheless, effectiveness of the program was not reported. Another study employed advance practice nurses to improve oral health of lowincome pregnant women in the United States. Although this program did not significantly improve women's knowledge, significant improvements in oral health practices and dental service uptake were reported, as well as a reduction in the rates of oral health problems in late pregnancy [47]. Oral health practices were also improved in Canada where maternal and child nutrition educators provided one-on-one dental preventive education to the whole community, including pregnant women [48]. Despite improvements in practice, the prevalence of ECC remained high.
Focus area 2: Antenatal oral health training programs for IHWs

Within focus area 2, three categories of literature were identified that could inform the training programs for IHWs: i) IHWs training programs to promote maternal oral health; ii) IHWs training programs to support oral health across the lifespan; and iii) training programs for non-dental health professionals in promoting maternal oral health.

\section{IHWs training programs to promote maternal oral health}

Mathu-Muju et al. [38] briefly described a Canadian government-initiated training program $(\mathrm{COHI})$ for IHWs to enable them to provide oral health education primarily for children, parents, caregivers, but also for expectant mothers. IHWs were trained in five areas not specifically defined in the study.

\section{IHWs training programs to support oral health across the lifespan}

This scoping review identified four Australian-based studies reporting different oral health training programs for IHWs to support Indigenous populations across the lifespan [43, $49,50]$. Two of the programs were introduced specifically to address the needs of communities in rural and remote areas [51]. Pacza et al. [51] in consultation with stakeholders from the local Indigenous medical service developed a three-module training program delivered in a classroom in addition to $60 \mathrm{~h}$ of on-the-job training. The modules covered an introduction to dental health and knowledge, applied oral health, and application to their local community. Another two studies delivered training through a one or 2 day workshop including education on oral health, oral hygiene and preventive treatments, emphasising the IHWs' utilisation of role-play, which received positive feedback [49, 50]. Smith et al. [50] also trained IHWs to use an early childhood oral health screening tool. Finally, one study [43] involved training IHWs to reduce ECC incidence through oral disease recognition, referral to dental services, oral health promotion, fluoride varnish application, provision of chart books and DVD instructions; however, mode of delivery and evaluation were not clearly described.

\section{Training programs for non-dental health professionals in promoting maternal oral health}

The search yielded four articles reporting training programs in promoting maternal oral health to non-dental health professionals including midwives $[44,52,53]$ and gynaecologists [54]. Two of these studies delivered faceto-face oral health education and promotion programs $[44,53]$ while the other two employed an online self-paced module [52] and written material [54] as the delivery mode of training activities. Öcek et al.s program in Turkey [53] 
increased oral health knowledge of midwives through a face-to-face program involving oral hygiene procedures, techniques, and eruption chronology of deciduous teeth in young children. Adams et al. [44] did not include an evaluation report, however, their face-to-face training in the United States for midwives included education on the importance of maternal oral health and dental care during pregnancy and instructions on oral health promoting practices including flossing and tooth-brushing, and was developed to be integrated into pre-existing antenatal sessions. George et al. [52] delivered and evaluated an online self-paced three-module program designed with theoretical and practical oral health content for midwives in Australia. The modules enhanced antenatal oral health knowledge, and also trained midwives to use an antenatal oral health screening tool which assisted their referrals to dental services. Deshpande et al. [54] also demonstrated an improvement in oral health knowledge with gynaecologists in India by means of a flipchart and oral health brochures.

\section{Focus area 3: Antenatal oral health screening tools developed for IHWs}

This scoping review could not identify any oral health screening tools developed specifically for IHWs in any setting. Nevertheless, there is one Australian report of IHWs being able to utilise an existing early childhood oral health screening tool, ubiquitously known as 'Lift the Lip', to identify ECC through a combination of parental concern and visual inspection [50]. IHWs could refer appropriately during a role-play demonstration, and reported the tool to be culturally acceptable. This resource has also been adapted for Australian Aboriginal and Torres Strait Islander families, and renamed as 'See My Smile' [55].

Five papers were identified that described an antenatal oral health screening tool for non-dental health professionals [22, 46, 56-58]. A descriptive study in the United States provided the 12 -item oral health questionnaire which was utilised by bilingual nurse midwives [57]. The questionnaire was assessed for cultural relevancy and currency by six pregnant women prior to its administration. The items identified the patient's health-seeking behaviours including dental service uptake, oral health status such as dental or gum pain, and risk factors such as vomiting frequency. Furthermore, a national consensus statement in the United States proposed a four item oral health questionnaire to be used by prenatal healthcare professionals [22]. This tool similarly aimed to identify oral health status, oral health risk factors and health-seeking behaviours. Another shorter screening tool developed for antenatal care providers, consisting of two items focusing on identifying oral health problems and visits to the dentist in the previous 6 months, was published in the New York State Department of Health [58] clinical guidelines. Stevens et al. [46] demonstrated that nurse midwives and nurse practitioners were able to utilise this screening tool, but no formal evaluation was conducted. An almost identical two-item screening tool assessing whether the pregnant woman had any dental problems, and whether they have visited a dentist in the last 12 months was developed and evaluated for midwives in Australia [56]. This tool was validated against two gold-standard assessments: a clinical evaluation by trained dentists and a subjective oral health assessment. It demonstrated high sensitivity in detecting women who required a dental visit.

\section{Discussion}

A review of the literature indicated that some IHWs have had roles in improving oral health outcomes of pregnant women, particularly in South Australia. However, there are currently no 'proof of concept' interventions, training programs or screening tools that can provide IHWs with the knowledge and skills to promote maternal oral health and refer pregnant women appropriately to dental services. While one study by MathuMuju et al. [38] trained IHWs to promote oral health among pregnant women, it focussed on reducing rates of ECC in children.

Although there is some evidence of maternal oral health training programs and screening tools available for nondental health professionals, certain issues require consideration prior to their use by IHWs. An important factor to consider is whether these interventions are culturally appropriate. Kreuter and colleagues [59] identified cultural appropriateness to be an essential element in interventions for Indigenous populations to reflect their unique health values, practices and behaviours. Another factor is that these programs were designed for individuals with a specific skill set to undertake oral assessments and provide oral health education. Consequently, there may be an incongruity between the educational background of IHWs and the clinical care requirements of these programs. For instance, some of the programs and tools were designed for midwives, nurses or other health professionals who typically complete tertiary level education [60]. However, in Australia, IHWs may not necessarily have the same degree of educational qualification as many complete vocational education only [61].

Despite this, a university-level education may not be a necessary pre-requisite to raise oral health awareness or to provide clinical care. In Australia, the United States and Canada, IHWs encouraged oral health within communities and played integral roles in applying fluoride varnish $[38,40,43,49]$. Various other studies have similarly demonstrated the key role of an IHW in breaking 
down barriers and bridging the gap in other healthcare settings. In the Australian state, Victoria, an Aboriginal liaison officer was the key contact through whom communities could choose to engage with the service [41]. They have also acted as a foundational support and conduit in the provision of care in mental health and cardiovascular settings where they developed mental health knowledge and assessment skills; improved Indigenous people's transition to mental health services; and more effectively delivered cardiovascular health education and care in hospitals with the expertise of other health professionals [62-64]. These studies highlight the capacity of IHWs to educate, assess and assist pregnant women to navigate through the healthcare system.

The literature also emphasises their role in brokering between communities and other healthcare professionals, including services with GPs, midwives, nurses and dentists. Walker and colleagues [65] explored the perspectives of health personnel, excluding IHWs, on the oral health role of IHWs. Their findings demonstrated that dental and nondental health personnel perceived IHWs to be in an important position to effectively promote oral health within their communities. Another study conducted with IHWs suggested that appropriate oral health training should be given by a team of IHWs and dentists to relay the relevant information to IHWs [66]. They also highlighted that training needed to be adopted by the team and that there should be adequate management support.

Models of care that enable effective partnerships between Indigenous women and IHWs, and that focus on continuity of care ensure that cultural needs are respected during this transition into parenthood. IHWs are integral to these partnerships as they share the same "language" as their community which facilitates effective communication [67]. This common language encourages compliance to interventions and uptake of available health services, including dental services, within the healthcare system $[29,68]$. Having the same language, fosters a relationship where patients feel a sense of empowerment and feel included in decision-making during the perinatal period, which further enhances self-efficacy [69]. Conway and colleagues [70] attributed the success of their program, which aimed to provide holistic, patient-centred chronic care management to Indigenous Australians, to its focus on the patient's story; providing patients the opportunity to make decisions about their own health.

Another important aspect in developing models of care in this area is to ensure the Aboriginal community is ready to receive oral health information from AHWs. One way of addressing this is to use motivational interviewing (MI). MI is a client-centred counselling intervention designed to elicit intrinsic behavioural change [71]. MI techniques have demonstrated significant effectiveness in improving health outcomes including blood pressure, cholesterol and body mass index [72].

Lastly, for effective transitional care and effective partnership models of care, there needs to be adequate support from the healthcare system. This may require organisational changes and sufficient resources and personnel to enact change [73, 74]. In Australia, free dental care generally is available through the public healthcare system for adults with low income. Although eligibility may not be conditional to Aboriginal and Torres Strait Islander status depending on the state, these individuals receive priority access [75]. Despite priority access, a high proportion of Aboriginal and Torres Strait Islander Australians do not regularly attend a dental service [76]. Campbell and colleagues [77] identified several barriers which can affect their access to health services which include long waiting times, distance, lack of transportation and previous experiences of racism. The lack of recruitment of Aboriginal and Torres Strait Islander staff in the healthcare system is another cited problem. It is recognised that to consolidate the Aboriginal health workforce requires specific support for the challenges they are likely to face including racism, community responsibilities, family issues, sense of isolation and stress and poor education [78]. To improve access, some Aboriginal Health Services, which provide free healthcare to their communities, are funded to provide dental care [75]. However, as not all Aboriginal Health Services receive funding for dental care, it is important to explore strategies to increase the number of Aboriginal and Torres Strait Islander dental staff and link their community to public dental services. The literature has shown great potential for capacity building IHWs to provide culturally-appropriate oral healthcare and referral to dental services within the healthcare system for pregnant women with adequate organisational support and resources.

There were a number of limitations in this review that need to be acknowledged. The strength of the evidence of the intervention studies which included IHWs were poor based on their study design quality [79] while the grey literature included may not necessarily be critically peerreviewed. Post-hoc analyses were frequently utilised in studies and few conducted significance testing. A possible explanation for this might be associated with the challenges of developing culturally-appropriate training programs and tools since considerable time may be necessary to negotiate and build partnerships with Indigenous communities [32]. There is also the ethical challenge of conducting rigorous research while ensuring that healthcare is not compromised for this vulnerable population. It was also difficult to define the role of IHWs from country to country which therefore limited comparisons. Further, there was a paucity of evidence comparing Indigenous populations with other disadvantaged groups, which may be a more useful comparison in identifying sociocultural barriers in future studies. 


\section{Recommendations}

Capacity building IHWs in maternal oral health requires both change to policy and practice. A structured framework incorporating organisational change and the allocation of sufficient resources may need to be implemented to strengthen oral health referral pathways from antenatal settings. Oral health interventions and protocols should be developed in partnership with IHWs to ensure training programs and tools are validated, pragmatic, culturally appropriate, easy to use, and accommodate continuity of care. Further, to address the needs of IHWs in both urban and remote areas, training programs may need to be designed to train-the-trainer or to adopt telehealth models.

\section{Conclusions}

IHWs over the globe have had some role in promoting oral health in the antenatal setting. Further work is necessary, however, to develop validated antenatal oral health training programs and screening assessment tools that respect Indigenous cultural values. More importantly, models of care that demonstrate and foster mutual partnerships that assist Indigenous peoples in accessing dental care require further research for implementation across the population.

\section{Abbreviations}

AHW: Aboriginal health worker; $\mathrm{COHI}$ : Children's Oral Health Initiative; ECC: Early childhood caries; IHW: Indigenous health worker; MeSH: Medical Subject Headings; $\mathrm{MIOH}$ : Midwifery initiated oral health; MIOH-DS: Midwifery initiated oral health dental service; $\mathrm{OH}$ : Oral health

\section{Acknowledgements \\ We would like to thank Mrs Melissa Burley for her assistance with the literature search.}

\section{Funding}

No financial support was received to develop this scoping review.

\section{Availability of data and materials}

Not applicable.

\section{Authors' contributions \\ $A G$ and $A C V$ were involved in the conception of the review and the development of the search strategy. ARV and ACV screened and reviewed the relevant articles. YS, AG, ARV, LMR, MSS, NJ, RS and ACV were involved in the interpretation and analysis of the data. All authors made substantial contribution to the drafting or revision of the manuscript for critically important intellectual content. All authors read and approved the final version to be published. Furthermore, all authors have agreed to be accountable for all aspects of the work in ensuring that questions related to the accuracy or integrity of any part of the work are appropriately investigated and seolved.}

Ethics approval and consent to participate

Not applicable.

\section{Consent for publication}

Not applicable.

\section{Competing interests}

The authors declare that they have no competing interests.

\section{Publisher's Note}

Springer Nature remains neutral with regard to jurisdictional claims in published maps and institutional affiliations.

\section{Author details}

${ }^{1}$ Centre for Oral Health Outcomes, Research Translation and Evaluation (COHORTE), Ingham Institute for Applied Medical Research, Locked Bag 7103, Liverpool BC, NSW 1871, Australia. ${ }^{2}$ School of Nursing \& Midwifery, Western Sydney University, Penrith 2751, Australia. ${ }^{3}$ South Western Sydney Local Health District, Liverpool, NSW, Australia. ${ }^{4}$ Ingham Institute for Applied Medical Research, Liverpool 1871, Australia. ${ }^{5}$ Centre for Applied Nursing Research (CANR), Liverpool 1871, Australia. ${ }^{6}$ Prince of Wales Clinical School, University of New South Wales, Sydney 2052, Australia. ${ }^{7}$ Faculty of Dentistry, University of Sydney, Camperdown 2050, Australia.

Received: 22 October 2017 Accepted: 8 March 2018

Published online: 20 March 2018

\section{References}

1. Anderson I, Robson B, Connolly M, Al-Yaman F, Bjertness E, King A, Tynan M, Madden R, Bang A, Coimbra CEA, et al. Indigenous and tribal peoples' health (the lancet-Lowitja Institute global collaboration): a population study. Lancet. 2016;388(10040):131-57.

2. King M, Smith A, Gracey M. Indigenous health part 2: the underlying causes of the health gap. Lancet. 2009;374(9683):76-85.

3. AlHW. The health and welfare of Australia's Aboriginal and Torres Strait Islander peoples 2015. Canberra: Australian Institute of Health and Welfare; 2015.

4. Chamberlain CR, Oldenburg B, Wilson AN, Eades SJ, O'Dea K, Oats JJN, Wolfe R. Type 2 diabetes after gestational diabetes: greater than fourfold risk among indigenous compared with non-indigenous Australian women. Diabetes Metab Res Rev. 2016;32(2):217-27.

5. Harris SB, Tompkins JW, TeHiwi B. Call to action: a new path for improving diabetes care for indigenous peoples, a global review. Diabetes Res Clin Pract. 2017;123:120-33.

6. AlHW. Birthweight of babies born to indigenous mothers. Canberra: Australian Institute of Health and Welfare; 2014.

7. Jamieson LM, Armfield J, Roberts-Thomson K. Oral health of aboriginal and Torres Strait islander children. Canberra: AlHW; 2007.

8. Martins-Júnior P, Vieira-Andrade R, Corrêa-Faria P, Oliveira-Ferreira F, Marques L, Ramos-Jorge M. Impact of early childhood caries on the oral health-related quality of life of preschool children and their parents. Caries Res. 2013:47(3):211-8.

9. Boggess KA, Edelstein BL. Oral health in women during preconception and pregnancy: implications for birth outcomes and infant oral health. Matern Child Health J. 2006;10(SUPPL. 7):169-74.

10. Kim Seow W. Environmental, maternal, and child factors which contribute to early childhood caries: a unifying conceptual model. Int J Paediatr Dent. 2012:22(3):157-68.

11. Klepacz-Szewczyk J, Pawlicka H. Most frequent oral pathological states problems occurring in pregnant patient. Dent Med Probl. 2014;51(3):387-96.

12. Silk H, Douglass AB, Douglass JM, Silk L. Oral health during pregnancy. Am Fam Physician. 2008;77(8):1139-44.

13. Tanwir F, Altamash M, Gustafsson A. Effect of diabetes on periodontal status of a population with poor oral health. Acta Odontol Scand. 2009;67(3):129-33.

14. Mascarenhas P, Fatela B, Barahona I. Effect of diabetes mellitus type 2 on salivary glucose - a systematic review and meta-analysis of observational studies. PLoS One. 2014;9(7):e101706.

15. Corbella S, Taschieri S, Del Fabbro M, Francetti L, Weinstein R, Ferrazzi E. Adverse pregnancy outcomes and periodontitis: a systematic review and meta-analysis exploring potential association. Quintessence Int. 2016;47(3): 193-204.

16. Kumar A, Basra M, Begum N, Rani V, Prasad S, Lamba AK, Verma M, Agarwal S, Sharma S. Association of maternal periodontal health with adverse pregnancy outcome. J Obstet Gynaecol Res. 2013;39(1):40-5.

17. Chapple ILC, Wilson NHF. Manifesto for a paradigm shift: periodontal health for a better life. Br Dent J. 2014;216(4):159-62

18. Yost J, Li Y. Promoting oral health from birth through childhood: prevention of early childhood caries. MCN Am J Matern Child Nurs. 2008;33(1):17-23.

19. Mitchell SC, Ruby JD, Moser S, Momeni S, Smith A, Osgood R, Litaker M, Childers N. Maternal transmission of mutans streptococci in severe-early childhood caries. Paediatr Dent. 2009;31(3):193-201. 
20. Jamieson LM, Parker EJ, Richards L. Using qualitative methodology to inform an indigenous-owned oral health promotion initiative in Australia. Health Promot Int. 2008;23(1):52-9.

21. Australian Health Ministers' Advisory Council. Clinical practice guidelines: antenatal care - module 1. Canberra: Australian Government Department of Health and Ageing; 2012

22. Oral Health Care During Pregnancy Expert Workgroup. Oral health care during pregnancy: a national consensus statement. Washington, DC: National Maternal and Child Oral Health Resource Center; 2012.

23. Jongen C, McCalman J, Bainbridge R, Aboriginal TK. Torres Strait islander maternal and child health and wellbeing: a systematic search of programs and services in Australian primary health care settings. BMC Pregnancy Childbirth. 2014:14(251):1-20.

24. Rumbold AR, Bailie RS, Si D, Dowden MC, Kennedy CM, Cox RJ, O'Donoghue L, Liddle HE, Kwedza RK, Thompson SC, et al. Delivery of maternal health care in indigenous primary care services: baseline data for an ongoing quality improvement initiative. BMC Pregnancy Childbirth. 2011:11(1):16.

25. AlHW. Australian burden of disease study: Impact and causes of illness and death in aboriginal and Torres Strait islander people 2011. Canberra: Australian Institute of Health and Welfare; 2016.

26. Weetra D, Glover K, Buckskin M, Kit JA, Leane C, Mitchell A, Stuart-Butler D, Turner M, Yelland J, Gartland D, et al. Stressful events, social health issues and psychological distress in aboriginal women having a baby in South Australia: implications for antenatal care. BMC Pregnancy Childbirth. 2016;16:88.

27. Wallace B, MacEntee M. Access to dental care for low-income adults: perceptions of affordability, availability and acceptability. J Community Health. 2012;37(1):32-9.

28. Department of Health and Ageing. Aboriginal Torres Strait islander health plan 2013-2023. Canberra: Department of Health and Ageing; 2013.

29. Abbott $\mathrm{P}$, Gordon E, Davison J. Expanding roles of aboriginal health workers in the primary care setting: seeking recognition. Contemp Nurse. 2008;27(2):157-64.

30. Bird M, Henderson C. Recognising and enhancing the role of aboriginal and Torres Strait islander health workers in general practice. Aborig $|s|$ Health Work J. 2005;29(3):32.

31. McDermott RA, Schmidt B, Preece C, Owens V, Taylor S, Li M, Esterman A. Community health workers improve diabetes care in remote Australian indigenous communities: results of a pragmatic cluster randomized controlled trial. BMC Health Serv Res. 2015;15(1):68.

32. Middleton P, Bubner T, Glover K, Rumbold A, Weetra D, Scheil W, Brown S. 'Partnerships are crucial': an evaluation of the aboriginal family birthing program in South Australia. Aust N Z J Public Health. 2017;41(1):21-6.

33. Stamp G, Champion S, Anderson G, Warren B, Stuart-Butler D, Doolan J, Boles C, Callaghan L, Foale A, Muyambi C. Aboriginal maternal and infant care workers: partners in caring for aboriginal mothers and babies. Rural Remote Health. 2008:8(883):6-8.

34. NSW Health. Definition of an aboriginal health worker, 2014. http://www1.health. nsw.gov.au/pds/ActivePDSDocuments/B2014 001.pdf. Accessed 10 Sep 2017.

35. Witmer A, Seifer SD, Finocchio L, Leslie J, O Neil EH. Community health workers: integral members of the health care work force. Am J Public Health. 1995;85(8):1055-8.

36. Arksey H, O'Malley L. Scoping studies: towards a methodological framework. Int J Soc Res Methodol. 2005;8(1):19-32.

37. South Australia Dental Service. Aboriginal oral health program: business plan. 2015. South Australia: SA Health.

38. Mathu-Muju KR, McLeod J, Walker ML, Chartier M, Harrison RL. The Children's oral health initiative: an intervention to address the challenges of dental caries in early childhood in Canada's first nation and Inuit communities. Can J Public Health. 2016;107(2):e188-93.

39. AlHW. Dental health of Indigenous children in the Northern Territory: Findings from the closing the gap program. Cat. No. IHW 41. Canberra: Australian Institute of Health and Welfare; 2011.

40. Braun PA, Quissell DO, Henderson WG, Bryant LL, Gregorich SE, George C, Toledo N, Cudeii D, Smith V, Johs N, et al. A cluster-randomized, community-based, tribally delivered oral health promotion trial in Navajo head start children. J Dent Res. 2016;95(11):1237-44.

41. DHSV. Dental services for Aboriginal and Torres Strait Islander Peoples: The Royal Dental Hospital of Melbourne. Victoria DHS: Victoria; 2017.

42. Parker E, Misan G, Richards L, Planning RA. Implementing the first stage of an oral health program for the Pika Wiya health service incorporated aboriginal community in Port Augusta, South Australia. Rural Remote Health. 2005;5(254):1-8.
43. Slade GD, Bailie RS, Roberts-Thomson K, Leach AJ, Raye I, Endean C, Simmons B, Morris P. Effect of health promotion and fluoride varnish on dental caries among Australian aboriginal children: results from a community-randomized controlled trial. Community Dent Oral Epidemiol. 2011;39(1):29-43.

44. Adams SH, Gregorich SE, Rising SS, Hutchison M, Chung LH. Integrating a nurse-midwife-led oral health intervention into CenteringPregnancy prenatal care: results of a pilot study. J Midwifery Womens Health. 2017; 62(4):463-9.

45. Johnson M, Ajwani S, Bhole S, Blinkhorn A, Dahlen H, Ellis S, George A. Promoting oral health in pregnant women through midwifery practice: an innovative approach. In: Australian nursing and midwifery conference. Newcastle: Newcastle City Hall; 2013. p. 2013.

46. Stevens J, lida $H$, Ingersoll G. Implementing an oral health program in a group prenatal practice. J Obstet Gynecol Neonatal Nurs. 2007:36(6):581-91.

47. Cibulka NJ, Forney S, Goodwin K, Lazaroff P, Sarabia R. Improving oral health in low-income pregnant women with a nurse practitioner-directed oral care program. J Am Acad Nurse Pract. 2011;23(5):249-57.

48. Lawrence $H$, Romanetz M, Rutherford L, Cappel L, Binguis D, Rogers JB. Oral health of aboriginal preschool children in northern Ontario. PRO. 2004;38(4): 172-90.

49. McGuire K, Tolentino R. Applied oral health workshop. Aborig Isl Health Work J. 1998;22(5):2-3.

50. Smith L, Blinkhorn F, Moir R, Brown N, Blinkhorn A. User assessment of an early childhood oral health education training course for aboriginal health workers. Int J Health Promot Educ. 2016;54(4):172-83.

51. Pacza T, Steele L, Tennant M. Development of oral health training for rural and remote aboriginal health workers. Aust J Rural Health. 2001:9(3):105-10.

52. George A, Lang G, Johnson M, Ridge A, de Silva AM, Ajwani S, Bhole S, Blinkhorn A, Dahlen HG, Ellis $\mathrm{S}$, et al. The evaluation of an oral health education program for midwives in Australia. Women Birth. 2016;29(3):208-13.

53. Öcek ZA, Eden E, Soyer MT, Cliçeklioglu M. Evaluation of a dental health education program for midwives. J Public Health Dent. 2003;63(4):255-7.

54. Deshpande AN, Dhillon SJ, Somanna KS, Dave BH, Porwal PA, Macwan CS. Impact of perinatal oral health care education programme on the knowledge, attitude and practice behavior amongst gynaecologists of Vadodara City. J Indian Soc Pedod Prev Dent. 2015;33(2):122-7.

55. COHS. See my smile. NSW: Centre for Oral Health Strategy; 2013.

56. George A, Dahlen HG, Blinkhorn A, Ajwani S, Bhole S, Ellis S, Yeo A, Elcombe E, Sadozai A, Johnson M. Measuring oral health during pregnancy: sensitivity and specificity of a maternal oral screening (MOS) tool. BMC Pregnancy Childbirth. 2016;16(347):1-6.

57. Hunter LP, Yount SM. Oral health and oral health care practices among lowincome pregnant women. J Midwifery Womens Health. 2011;56(2):103-9.

58. New York State Department of Health. Oral health care during pregnancy: practice guidelines. New York: NYSDOH; 2006

59. Kreuter MW, Lukwago SN, Bucholtz DC, Clark EM, Sanders-Thompson V. Achieving cultural appropriateness in health promotion programs: targeted and tailored approaches. Health Educ Behav. 2003;30(2):133-46.

60. Lusk B, Russell RL, Rodgers J, Wilson-Barnett J. Preregistration nursing education in Australia, New Zealand, the United Kingdom, and the United States of America. J Nurs Educ. 2001;40(5):197-202.

61. Rose M, Pulver LRJ. Aboriginal health workers: professional qualifications to match their health promotion roles. Health Promot J Austr. 2004;15(3):240-4.

62. Aoun S, Johnson L. Capacity building in rural mental health in Western Australia. Aust J Rural Health. 2002;10(1):39-44

63. McKenna B, Fernbacher S, Furness T, Hannon M. "cultural brokerage" and beyond: piloting the role of an urban aboriginal mental health liaison officer. BMC Public Health. 2015;15:881.

64. Taylor KP, Thompson SC, Smith JS, Dimer L, Ali M, Wood MM. Exploring the impact of an aboriginal health worker on hospitalised aboriginal experiences: lessons from cardiology. Aust Health Rev. 2009;33(4):549-57.

65. Walker D, Tennant M, Short SD. An exploration of the priority remote health personnel give to the development of the indigenous health worker oral health role and why: unexpected findings. Aust J Rural Health. 2013;21(5): 274-8.

66. Walker D, Tennant M, Short SD. Listening to indigenous health workers: helping to explain the disconnect between policy and practice in oral health role development in remote Australia. Health Educ J. 2011;70(4):400-6.

67. Giblin PT. Effective utilization and evaluation of indigenous health care workers. Public Health Rep. 1989;104(4):361. 
68. Andrews B, Simmons P, Long I, Identifying WR. Overcoming the barriers to aboriginal access to general practitioner services in rural new South Wales. Aust J Rural Health. 2002;10(4):196-201.

69. Sørensen K, Van den Broucke S, Fullam J, Doyle G, Pelikan J, Slonska Z, Brand $\mathrm{H}$. Health literacy and public health: a systematic review and integration of definitions and models. BMC Public Health. 2012;12(1):80.

70. Conway J, Tsourtos G, Lawn S. The barriers and facilitators that indigenous health workers experience in their workplace and communities in providing self-management support: a multiple case study. BMC Health Serv Res. 2017;17(1):319.

71. Miller WR. Motivational interviewing with problem drinkers. Behav Cogn Psychother. 1983;11(2):147-72.

72. Rubak S, Sandbæk A, Lauritzen T, Christensen B. Motivational interviewing: a systematic review and meta-analysis. Br J Gen Pract. 2005;55(513):305-12.

73. Andrews S, Austin N, Clarke A, Goodman H, Miller J. Promoting koori Children's health - an affirmative approach. Health Promot J Austr. 1998;8(1): 29-33.

74. Cheng M, Clarke A, Moore T, Lau P. Making the connection: a qualitative study of brokerage in aboriginal health in a metropolitan area of Victoria and a regional area of new South Wales. Aust Health Rev. 2011;35(1):18-22.

75. Schwarz E. Access to oral health care - an Australian perspective. Community Dent Oral Epidemiol. 2006;34(3):225-31.

76. Amarasena N, Kapellas K, Skilton MR, Maple-Brown L, Brown A, Bartold M, O'Dea K, Celermajer D, Jamieson LM. Factors associated with routine dental attendance among aboriginal Australians. J Health Care Poor Underserved. 2016:27(1):67-80.

77. Campbell MA, Hunt J, Walker D, Williams R. The oral health care experiences of NSW aboriginal community controlled health services. Aust N Z J Public Health. 2015;39(1):21-5.

78. Gwynne K, Lincoln MP. Developing the rural health workforce to improve Australian aboriginal and Torres Strait islander health outcomes: a systematic review. Aust Health Rev. 2017;41 (2):234-8.

79. Merlin T, Weston A, Tooher R. Extending an evidence hierarchy to include topics other than treatment: revising the Australian 'levels of evidence'. BMC Med Res Methodol. 2009;9(1):34.

\section{Submit your next manuscript to BioMed Central and we will help you at every step:}

- We accept pre-submission inquiries

- Our selector tool helps you to find the most relevant journal

- We provide round the clock customer support

- Convenient online submission

- Thorough peer review

- Inclusion in PubMed and all major indexing services

- Maximum visibility for your research

Submit your manuscript at www.biomedcentral.com/submit

) Biomed Central 